\title{
Umbilical cord stem cells: biological characteristics, approaches to banking and clinical application
}

\author{
Nasadyuk C. M. \\ Institute of Cell Therapy, Kyiv, Ukraine \\ e-mail: nasadyukch@gmail.com
}

\section{ABSTRACT}

Due to the prominent immunosuppressive and regenerative properties, umbilical cord mesenchymal stem cells are the most widely explored in the treatment of autoimmune diseases and posttransplant complications as well as for the facilitation of engraftment of hematopoietic stem cell transplant and cell culturing in vitro. The review presents modern immunophenotypic characterization of the umbilical cord mesenchymal stem cells, approaches to isolation, biobanking, and clinical application.

KEYWORDS: umbilical cord; mesenchymal stem cells; cell therapy; biobanking

Umbilical cord is a unique anatomical structure, that connects the maternal and fetus organisms during pregnancy, provides fetus with oxygen, nutrients and excrets the products of its life-sustaining activity. Umbilical cord contains 2 umbilical arteries and 1 vein, covered by a special type of the mucous connective tissue, called Wharton's jelly, which in its turn is covered by the amniotic epithelium [1].

After childbirth the function of the umbilical cord is supposed to be performed and during many years the umbilical cord together with the placenta were utilized. From the end of 80 s of the 20 th century the attitude to the umbilical cord changed since it was found that blood, remaining in umbilical vessels contains a significant number of hematopoietic stem cells, which are increasingly used in clinical practice as a bone marrow alternative [2, 3]. Up-to-date more than 35,000 umbilical cord blood hematopoietic stem cells transplants were performed worldwide for the treatment of malignant and other diseases in children and adults and cord blood banks occupied an important place in the system of health care institutions [2].

In 1991, umbilical cord tissue was accepted in the list of the potential stem cells sources for the purposes of tissue engineering, when the group McElreavey et al. for the first time isolated fibroblast-like cells from the Wharton's jelly of human umbilical cord, which are the type of mesenchymal stem cells (MSCs) $[1,4,5]$. Human MSCs are also being isolated from the bone marrow, umbilical cord blood, adipose tissue, amniotic fluid, endometrium, dental tissues etc. [3, 6, 9].

Umbilical cord stem cells may be considered to be mesenchymal since they match minimal criteria, determined in 2006 by the Mesenchymal and Tissue Stem Cell Committee of the International Society for Cellular Therapy [50]. Hence, umbilical cord MSCs were shown to express surface markers CD29, CD44, CD73, CD90, CD105 and do not express CD14, CD34, CD45 i HLA-DR [8, 50]. Umbilical cord mesenchymal stem cells are characterized by low expression of MHC I class antigens, what protects them from the attack of the natural killer cells of the recipients after allogeneic application as well as low or zero expression of MHC II class antigens [8]. This allows to consider the umbilical cord stem cells less immunogenic compared to cells, isolated from the other sources.

Umbilical cord MSCs are capable to self-renewal and pluripotency and can differentiate not only into mesenchymal (osteocytes, adipocytes, chondrocytes) but also non-mesenchymal cell lines, including derivatives of ectoderm (astrocytes, oligodendrocytes, neurons) and endoderm hepatocytes $[1,8,9]$. The possibility of differentiation of umbilical cord stem cells into cardiomyocytes, osteocytes, chondrocytes, myocytes, adipocytes etc. was also shown [12]. Proliferative potential of the umbilical cord mesenchymal stem cells overexceeds clonogenic potential of the analogous cells of menstrual blood and adipose tissue [9]. The results of studies on pluripotency of the umbilical cord MSCs create predispositions for their clinical application in regenerative medicine. Due to phenotype and biological features of the umbilical cord mesenchymal stem cells, the latter are considered to be a "bridge" between embryonic and adult stem cells $[8,9]$.

As a potential source of the different populations of stem cells the following parts of the umbilical cord possess value: Wharton's jelly, intravascular zone, perivascular zone, subamniotic zone, blood vessels, amniotic epithelium [1,11, 12]. Mesenchymal stem cells are isolated from the Wharton's jelly, whereas epithelial cells - from the internal and external layers of the umbilical cord $[1,11]$. Endothelial progenitor cells, perspective for the purposes of tissue engineering of the vessel constructions, may be isolated from the tissues, surrounding Wharton's jelly as well as umbilical veins and artery [1,12]. Umbilical cord MSCs were shown to produce such important biologically active compounds as intereukin-10, transforming growth factor beta, interleukin-6, vascular endothelial growth factor, prostaglandin E2, cyclooxygenases, hepatocyte growth factor, leukemia inhibiting factor, indoleamine 2,3-dioxygenase [6]. 
Today two methods are used for the isolation of mesenchymal stem cells from the umbilical cord: enzymatic treatment of the tissue using collagenase I, trypsin, hyaluronidase and explants technique $[1,7,10$, $11,14]$.

The advantages of the umbilical cord stem cells compared to MSCs, derived from the other sources of the adult organism, are:

- Availability, cost-effectiveness, ethics and simplicity of obtaining $[1,4,13]$;

- Higher potential of expansion and differentiation compared to other adult stem cells [14];

- Lower risk of infections transmission [1];

- Lower risk of teratoma development in clinical use [1];

- Multipotency [1, 2];

- Low immunogenicity $[1,6]$;

- Immunosuppressive effect [1, 24, 25, 46];

- Stimulation of hematopoietic stem cells engraftment in combined use [13, 32, 33, 34];

- Effective medium for the culture of other cell populations [6, 14];

- Valuable cellular raw material for the needs of tissue engineering [10].

\section{WORLD EXPERIENCE OF UMBILICAL TISSUE BANKING}

Storage of the umbilical cord tissue enjoys increasing popularity worldwide and up-to-date such service is offered by the majority of cord blood banks of a family type together with the storage of the umbilical cord blood [2, 3]. This service is marketed as an opportunity to get a unique source of MSCs [1]. In some countries of the world preservation of the umbilical cord simultaneously with the umbilical cord blood is chosen by about $70 \%$ families.

Storage of the umbilical cord implicates 2 technological approaches: isolation and cryopreservation of mesenchymal stem cells as a ready off-the-shelf GMP-product and/or storage of the piece of the tissue with the perspective of isolation of cell population in future, in case of the necessity of its clinical application $[5,12,15,16]$. The latter is supported by the arguments on the fast development of the science and evidence of the discovery of the new, unknown today, stem cells populations, which it will be possible to isolate from the cryopreserved tissue. At the same time literary data provides evidence that isolation of stem cells from the umbilical cord before cryopreservation increases 8-fold the number of cells that may be preserved [15]. Some biobanks offer storage of anatomically different parts of the umbilical cord, that can contain different populations of the stem cells [1,4].

There is no explicit opinion about the volume of the umbilical cord tissue that should be stored. Some biobanks encourage the obstetricians to collect as long as possible piece of the umbilical cord although more often $12-15 \mathrm{~cm}$ are stored. Confident expansion of umbilical cord tissue banking in world bioeconomics is confirmed by the commercial offers of numerous containers for the transportation of this kind of biomaterial and other supplies for its processing.

In European countries the cost of the storage of the umbilical tissue is usually 2-fold lower than cryopreservation of the umbilical cord blood, whereas in the countries of the region of Asia and Oceania the cost of these services is balanced. Storage of the umbilical cord in the arsenal of services of the biobank is believed to make it more competitive [16].

\section{APPROACHES TO CLINICAL APPLICATION OF THE UMBBILICAL}

\section{CORD STEM CELLS}

Prominent regenerative and immunosuppressive properties of mesenchymal stem cells of the umbilical cord assumpt their increasing application in biomedical research and clinical practice $[4,12,17,20,23$, 33]. As of 2015 , more than 50 clinical trials, devoted to the evaluation of the safety and efficacy of the umbilical cord stem cells in the treatment of various diseases were registered, i.e. knee osteoarthritis, rheumatic arthritis, autism, bronchial asthma, burns, infertility, malignant blood disorders [17]. In general, introduction of the human umbilical cord
MSCs into practical medicine and biotechnologies occurs in the following directions:

- The treatment of autoimmune disorders (rheumatic arthritis, multiple sclerosis, ulcerative colitis, Crohn's disease etc.) [18, 19, 24, 45];

- The treatment of posttransplant immune complications and facilitation of engraftment of hematopoietic stem cells of the bone marrow, peripheral or cord blood [31, 32, 33, 34];

- The treatment of cardiovascular diseases (post-infarction cardiosclerosis, cardiomyopathy, ischemic heart disease, arrhythmia) [37, 38];

- The treatment of liver cirrhosis and liver failure [39, 40, 41];

- The treatment of musculoskeletal system disorders (osteoarthritis, traumatic disorders of joints and tendons, sports trauma, congenital or genetically mediated bones disorders) [7, 35];

- The treatment of nervous system disorders [45, 46, 47];

- The treatment of diabetes mellitus [20];

- Bioengineering of tissues and organs (skin and vessels equivalents, heart valves, bone and cartilage tissue constructs) [7, 10, 35];

- In laboratory practice and biotechnological industry as a feeder for culture of hematopoietic and other stem cells [14].

\section{APPLICATION OF THE UMBILICAL CORD MSCS}

\section{IN THE TREATMENT OF AUTOIMIMUNE DISEASES}

Today it is accurately known that MSCs, including the ones derived from the human umbilical cord, possess immunosuppressive effect, what makes them a perspective remedy in the treatment of autoimmune disorders and posttransplant complications $[18,19]$. In vivo and in vitro studies showed the interaction between mesenchymal stem cells with regulatory T-lymphocytes and in vitro inhibition of proliferation and cytotoxicity of natural killer and dendritic cells, decrease of the production of cytokines, antibodies, processes of antigen presentation [18, 21, 22, 23], i.e. inhibition of the mechanisms of immune pathology development.

Rheumatoid arthritis is the most prevalent autoimmune disorder and despite the achievements of modern pharmacology, efficacy of treatment of this disease demands the development of new alternative therapies $[19,24]$. Initiation of clinical trials on the evaluation of safety and efficacy of cell therapy in the treatment of rheumatoid arthritis was preceded by the positive results of experimental studies, in which mesenchymal stem cells derived from the bone marrow, synovial fluid and umbilical cord were used [19, 24].

In the clinical trial, encompassing 172 patients with the active form of the rheumatoid arthritis with proved uneffectiveness of the conventional treatment, therapy with antirheumatic drugs was conducted simultaneously with the introduction of umbilical cord MSCs and on the background of placebo instead of the administration of the cell preparation. Dose of the umbilical cord MSCs made $4 \cdot 10^{7}$ cells per one intravenous infusion. Adverse effects of the cell therapy were not revealed. The use of the umbilical cord MSCs resulted in the decrease of the level of tumor necrosis factor-alpha in blood; also in the peripheral blood the increase of the percentage of $\mathrm{CD}^{+}{ }^{+} \mathrm{CD} 25^{+} \mathrm{Foxp} 3^{+}$regulatory T-lymphocytes was noted. Due to the American College of Rheumatology improvement criteria and health survey, therapeutic effect of cell therapy lasted 3-6 months and was continued by the repeated infusion of the mesenchymal stem cells [24].

System inflammatory disorders of the colon, i.e. Crohn's disease and ulcerative colitis hold an important place among autoimmune diseases, the treatment of which includes application of stem cells. In experimental studies in mice with dextran sulfate sodium (DSS)-induced colitis intraperitoneal administration of the umbilical cord MSCs was shown to decrease the signs of inflammation in the mucous membrane of the colon, also regulatory effect of these cells on the immune reactions in spleen and mesenterial lymphatic nodes was shown [25]. Systemic or local therapy with mesenchymal stem cells was carried out in more than 1,500 patients with Crohn's disease and ulcerative colitis worldwide [18]. Actually cell therapy is regarded as the next generation of the therapeutic measures in 
the treatment of systemic inflammatory diseases of the colon, especially in patients with secondary resistance to anti-cytokine therapy $[18,26]$.

Application of the MSCs in systemic inflammatory disorders of the colon was shown to decrease pain syndrome, diarrhea and bleeding and to stimulate reparation processes $[25,26,27,28]$. The first and second phases of clinical trials proved the safety of mesenchymal stem cells administration in the treatment of Crohn's disease and ulcerative colitis and their advantages compared to the use of fibrin glue in fistulizing colitis $[18,27,29]$.

The umbilical cord MSCs were also shown to be safe and effective in the treatment of lupus erythematosus. Hence, results of treatment of 40 patients with lupus erythematosus showed that this therapy was well tolerated by the patients and had positive therapeutic effect, although 6 months later in some patients cell therapy course was repeated because of the relapse of the disease [30].

\section{APPLICATION OF THE UMBILICAL CORD MSCS IN THE TREATMENT} OF IMMUNOLOGIC POSTTRANSPLANT COMPLICATIONS

AND FOR THE FACILITATION OF ENGRAFTMENT OF HEMATOPOIETIC STEM CELLS TRANSPLANT

Introduction of the mesenchymal stem cells belongs to important therapeutic strategies in hematologic transplantology. Adjuvant transplant of umbilical cord MSCs was shown to facilitate engraftment of the transplant of the bone marrow, peripheral and cord blood [31, 32, 33 , 34]. Due to outstanding immunosuppressive properties umbilical cord mesenchymal stem cells are also increasingly used in the treatment of the graft-versus-host disease [51].

Experimental research on mice showed that MSCs expressing SDF1/HOXB4 significantly enhance the growth of the hematopoietic stem cells in vitro and their engraftment in vivo. Therapy with these cells significantly increased of survival and hematopoiesis reconstitution in lethally irradiated animals after hematopoietic stem cells transplantation $[31,36]$.

In a clinical trial 20 patients with a high risk leukemia were randomly divided into two groups: the $1^{\text {st }}$ group was performed transplant of cord blood and ex vivo expanded umbilical cord MSCs, whereas the patients of the $2^{\text {nd }}$ group were transplanted only cord blood. Period of engraftment of the neutrophils and platelets was significantly shorter in 8 patients, administered umbilical cord mesenchymal stem cells compared to indices in 12 patients, who received only cord blood transplant [33]. No serious adverse effects of umbilical cord MSCs treatment were noted [33].

\section{THE APPLICATION OF THE UMBILICAL CORD MSCS}

\section{IN THE TREATMIENT OF CARDIOVASCULAR DISEASES}

Production of numerous growth factors by the umbilical cord MSCs as well as their ability to differentiate into cardiomyocytes, endotheliocytes and other cell lines mediate the use of this cell population in the treatment of cardiovascular diseases (post-infarction cardiosclerosis, coronary artery disease, atherosclerosis, chronic limbs ischemia, cardiomyopathy, Burger's disease, arrhythmia etc. [10, 37, 38].

At least 67 clinical trials on the evaluation of the efficacy of mesenchymal stem cells in the treatment of heart disorders and about 8 trials on the treatment of vessels are registered in the international database of clinical trials. Among them in 11 trials the umbilical cord MSCs are used for the treatment of vascular diseases and in 7 trials - for the treatment of heart, mainly cardiomyopathy [17].

On the experimental model of the myocardial infarction the umbilical cord MSCs and endothelial progenitors derived from them were shown to enhance regeneration of the infarcted heart, to stimulate processes of vascularization and decrease fibrosis. The scientists explain the regenerative effect of the umbilical cord MSCs in the therapy of the post-infarction cardiosclerosis by humoral mechanisms, in particular the production of a variety of growth factors by these cells [37].

The efficacy of the umbilical cord mesenchymal stem cells was investigated in the treatment of a systolic type heart failure. 59 patients were randomly divided into 2 groups: the experimental group was administered pharmacotherapy simultaneously with intracoronary transplant of the umbilical cord MSCs, whereas the control group (29 patients) received only pharmacotherapy. One month after the cell therapy the level of fatigue, chest discomfort and shortness of breath remained high but the 6-min walk distance was significantly longer in individuals, receiving the umbilical cord MSCs compared to patients treated only by pharmacotherapy. The ejection fraction in the group of patients, administered cell therapy, also was higher compared to the control group and level of mortality was lower [38].

\section{THE APPLICATION OF THE UMBILICAL CORD MSCS IN THE}

TREATMENT OF LIVER CIRRHOSIS AND LIVER FAILURE

Cell therapy is increasingly used in the treatment of hepatobiliary system diseases. Experimental studies showed the ability of the umbilical cord MSCs to differentiate into hepatocyte-like cells under the effect of the liver extract of the rat with induced fibrosis [39]. Cells obtained from the umbilical cord mesenchymal stem cells were characterized by hepatocytes biomarkers AFP, CK18 and CYP3A4 and were able to perform hepatocyte-specific functions, in particular to glycogen storage, produce urea and albumin [39].

Another but clinical study, encompassing 50 patients, showed that intrahepatic transplant of the umbilical cord MSCs to patients with decompensated liver cirrhosis resulted in decrease of the edema and oliguria during the first 2-3 weeks after the conducted therapy. Simultaneously the patients received hepatoprotective, antiviral therapies as well as other conventional drugs. During $24 \mathrm{~h}$ period no adverse effects of the umbilical cord mesenchymal stem cells transplant were revealed. The significant increase of the level of albumin and prealbumin in blood serum was noted. At the same time due to ultrasound examination changes in hemodynamics of the portal and spleen veins were not found, whereas the level of alpha-fetoprotein and blood coagulation factors did not change significantly [40].

The possibility of differentiation of the umbilical cord MSCs into hepatocytes was shown also in other papers what assume their broader application in the treatment of disorders accompanied by liver failure [41].

\section{THE APPLICATION OF THE UMBILICAL CORD MSCS IN THE \\ TREATMENT OF THE MUSCULOSKELETAL DISORDERS}

Due to high potential of the mesenchymal stem cells to osteogenic and chondrogenic differentiation, one of the first directions of their application in regenerative medicine was the treatment of disorders and injuries of the musculoskeletal system (osteoarthritis and osteoarthrosis, bone fractures, sports injuries, rheumatoid arthritis etc.) as well as in tissue engineering of the bone and cartilage tissue constructs for reconstructive orthopedic surgery $[7,8,35,36,43]$. The umbilical cord mesenchymal stem cells were shown to be capable to migration to joints affected by the pathologic process and enhance their regeneration [42]. The involvement of the circulating progenitor cells in regeneration of the damaged bone was shown by scientists [43], although as of today the molecular mechanisms of homing of these cells in the foci of injury are not elucidated as well as their proliferation and differentiation into the bone tissue.

\section{THE APPLICATION OF THE UMBILICAL CORD MSCs}

\section{IN THE TREATMENT OF DIABETES MELLITUS}

Due to modern knowledge the development of type 1 diabetes mellitus is mostly associated with the destruction of the insulin-producing beta cells in islets of Langerhans by autoantibodies [20]. Hence, modern approaches to the search of the alternative to insulin therapy methods of treatment of type 1 diabetes are based on the inhibition of autoimmune aggression on early stages of the disease, what may prevent the destruction of remained beta-cells [20]. Due to immunosuppressive properties of the mesenchymal stem cells, cell therapy is regarded as a perspective method of treatment of the type 1 diabetes mellitus. And the ability of umbilical cord MSCs to produce numerous growth factors as 
well as multilinear transdifferentiation allow to use them in the treatment of complications of diabetes mellitus type 2 (ischemia of lower limbs, retinopathy, nephropathy) [6].

The results of foreign studies showed that the umbilical cord blood MSCs transplant to patients with type 2 diabetes allowed to stop insulin therapy in half of the patients for a period of 25-43 months and to decrease its dose in the rest of the patients. Increase of the level of C-peptide during the first month after the treatment, lasting for the whole 24 month followup period was also noted. In all patients, who were transplanted the umbilical cord MSCs the level of glucose in fasting condition and $2 \mathrm{~h}$ after meals remained stable. Toxic effects of the therapy by the umbilical cord MSCs in type 2 diabetes mellitus patients were not found [20].

\section{APPLICATION OF THE UMBILICAL CORD MSCS IN THE TREATMENT}

\section{OF THE NERVOUS SYSTEM DISORDERS}

Regenerative potential of the umbilical cord MSCs is used also in the treatment of the nervous system disorders: Parkinson's disease, Alzheimer's disease, stroke, injury of the brain and spinal cord. Although functional differentiation and integration of the neural cells obtained from the MSCs is not yet proved in vivo, neuroprotective effect of cell therapy by means of the production of various neurotrophic factors is obvious [45]. Due to the literary data, the umbilical cord may serve as a source of the mesenchymal stem cells for the clinical application in neurology $[1,4$, 45]. The safety and efficacy of the application of the umbilical cord MSCs in the treatment of the multiple sclerosis was shown [45]. Efficacy of the therapy with the umbilical cord MSCs of thoracolumbar injury was also reported [46].
On the model of the experimental injury of the cavernous nerve in rats, the injection of human umbilical cord MSCs, producing brainderived neurotrophic factor (BDNF) into penis was shown to enhance regeneration of the cavernous nerve, to decrease fibrosis of corpora cavernosa and improve erectile function [47].

\section{APPLICATION OF THE UMBBILICAL CORD MISCS IN BIOENGINEERING} OF TISSUES AND ORGANS

Umbilical cord MSCs are treated as the initial biological product for the bioengineering of tissues and organs since they can differentiate not only into mesenchymal (osteocytes, adipocytes, chondrocytes) but also nonmesenchymal cell lines, including derivatives of ectoderma (astrocytes, oligodendrocytes, neurons) and endoderma (hepatocytes) [1, 8].

The search for the optimal substitute material for the reconstruction of bone tissue, avoiding immunologic complications, inflammation, distortion is a hot issue in modern traumatology. In this direction nonimmunogenic stem cells with chondrogenic potential, including the umbilical cord MSCs, are increasingly investigated.

In recent years cell therapy also finds application in dental implantology: the mesenchymal stem cells are used for the growth of bone before installation of implants [6]. Technologies of development of bioprosthesis of heart valves and vessel grafts as well as pieces of skin from the mesenchymal stem cells of different sources are being worked out $[7,10,43]$.

Another niche for the application of the umbilical cord MSCs is their use as a feeder for culture of the hematopoietic and other stem cells [4, $12,14]$.

\title{
CONCLUSIONS
}

\begin{abstract}
Presented data state that umbilical cord is a valuable source of the stem cells for the clinical application and an important object of the biobanking. American Association of Blood Banks (AABB) approved accreditation standards on somatic cells banking, which regulate also the isolation of cells from the umbilical cord tissue [18]. In Ukraine, Institute of Cell Therapy developed protocols of cultivation of the umbilical cord mesenchymal stem cell, directed at the production of the high-quality off-the-shelf GMP-product.
\end{abstract}

\section{REFERENCES}

1. Nagamura-Inoue T, He H. Umbilical cord-derived mesenchymal stem cells: Their advantages and potential clinical utility. World J Stem Cells. 2014; 6(2):95-202.

2. Nasadyuk CM, Makhinya AV, Zak SO, et al. Current status and perspectives of cryostorage and clinical application of the umbilical cord blood in Ukraine and in the World. Cell and organ Transplantology. 2015; 3(2):214-219.

3. Overview of the World congress on cord blood and innovative approaches to the treatment of sickle-cell anemia in Monaco on 24-27 October 2013. Cell and organ Transplantology. 2014; 2(1):95-101.

4. Ding DC, Chang YH, Shyu WC, et al. Human Umbilical Cord Mesenchymal Stem Cells: A New Era for Stem Cell Therapy. Cell Transplant. 2015; 24(3):339-347.

5. Cooper K, Shah V, Sapre N, et al. Defining Permissible Time Lapse between Umbilical Cord Tissue Collection and Commencement of Cell Isolation. Int J Hematol Oncol Stem Cell Res. 2013; 7(4):15-23.

6. Review of reports of the $13^{\text {th }}$ meeting of the International Society for Stem Cell Research - ISSCR (24-27 June 2015, Stockholm, Sweden). Cell and organ Transplantology 2015; 3(2):224-26

7. Petrou IG, Grognuz A, Hirt-Burri N, et al. Cell therapies or tendons: old cell choice for modern innovation. Swiss Med Wkly. 2014; 7:144:w13989.

8. Ullah I, Subbarao RB, Gyu JR. Human mesenchymal stem cells - current trends and future prospective. Biosci Rep. 2015; 35(2):e00191.

9. Chen JY, Mou XZ, Du XC, et al. Comparative analysis of biological characteristics of adult mesenchymal stem cells with different tissue origins. Asian Pac J Trop Med. 2015; 8(9):739-46.

10. Bourget JM, Gauvin R, Duchesneau D, et al. Potential of Newborn and Adult Stem Cells for the Production of Vascular Constructs Using the Living Tissue Sheet Approach. Biomed Res Int. 2015; 2015:168294.

11. Cardoso TC, Ferrari HF, Garcia AF. Isolation and characterization of Wharton's jelly-derived multipotent mesenchymal stromal cells obtained from bovine umbilical cord and maintained in a defined serum-free three-dimensional system. BMC Biotechnol. 2012; 4(12):P18.

12. Polchow $B$, Kebbel $K$, Schmiedeknecht $G$, et al. Cryopreservation of human vascular umbilical cord cells under good manufacturing practice conditions for future cell banks. J Transl Med. 2012; 16(10):98.

13. Wang L, Weiss ML, Detamore MS. Recent Patents Pertaining to Immune Modulation and Musculoskeletal Regeneration with Wharton's Jelly Cell. Recent Pat Regen Med. 2013; 3(3):182-92

14. Kadekar D, Kale V, Limaye L. Differential ability of MSCs isolated from placenta and cord as feeders for supporting ex vivo expansion of umbilical cord blood derived CD34(+) cells. Stem Cell Res Ther. 2015; 19(6):201. 
15. Briddell R, Litkenhaus $F$, Foertsch $G$, et al. Recovery of viable MSCs isolated from fresh umbilical cord tissue, measured after cryopreservation, is on average 8-fold higher when compared to recovery of viable MSCs isolated from previously cryopreserved umbilical cord tissue. Blood (ASH Annual Meeting Abstracts). 2011; 118: Abstract 4398.

16. http://parentsguidecordblood.org

17. www.clinicaltrials.gov

18. Nasadyuk CM. Stem Cell Therapy In The Treatment Of Inflammatory Bowel Disease. Gastroenterology\&Hepatology: Open Access. 2014; 1(1):00006.

19. El-Jawhari JJ, El-Sherbiny YM, Jones EA, et al. Mesenchymal stem cells, autoimmunity and rheumatoid arthritis. QJM. 2014; 107(7):505-14.

20. Li-Xue G, Hui G, hai-Bo L, et al. Therapeutic efficacy of umbilical cord-derived mesenchymal stem cells in patients with type 2 diabetes. Experimental and Therapeutic Medicine. 2015; 9:1623-30.

21. Zuo D, Liu X, Shou Z, et al. Study on the interactions between transplanted bone marrow-derived mesenchymal stem cells and regulatory $T$ cells for the treatment of experimental colitis. Int J Mol Med. 2013; 32(6):1337-44.

22. de Miguel MP, Fuentes-Julián S, Blázquez-Martínez A, et al. Immunosuppressive properties of mesenchymal stem cells: advances and applications. Curr Mol Med. 2012; 12(5):574-91.

23. Hayashi Y, Tsuji S, Tsuji M, et al. Topical implantation of mesenchymal stem cells has beneficial effects on healing of experimental colitis in rats. J Pharmacol Exp Ther. 2008; 326(2):523-31.

24. Wang L, Cong X, Liu G, et al. Human umbilical cord mesenchymal stem cell therapy for patients with active rheumatoid arthritis: safety and efficacy. Stem Cells Dev. 2013; 22(24):3192-3202.

25. Li L, Liu S, Xu Y, et al. Human umbilical cord-derived mesenchymal stem cells downregulate inflammatory responses by shifting the Treg/Th17 profile in experimental colitis. Pharmacology. 2013; 92(5-6):257-64.

26. Nasadyuk CM. Cell Therapy in Gastroenterology. Cell and Organ Transplantology. 2015; 3(1):78-81.

27. Voswinkel J, Francois $S$, Gorin NC, et al. Gastro-intestinal autoimmunity: preclinical experiences and successful therapy of fistulizing bowel diseases and gut graft versus host disease by mesenchymal stromal cells. Immunol Res. 2013; 56(2-3):241-48.

28. Deng $X$, Szabo S, Chen L, et al. New cell therapy using bone marrow-derived stem cells/endothelial progenitor cells to accelerate neovascularization in healing of experimental ulcerative colitis. Curr Pharm. Des. 2011; 17(16):1643-51.

29. van Deen WK, Oikonomopoulos A, Hommes DW. Stem cell therapy in inflammatory bowel disease: which, when and how. Curr Opin Gastroenterol. 2013; 29(4):384390 .

30. Wang $D$, Li J, Zhang $Y$, et al. Umbilical cord mesenchymal stem cell transplantation in active and refractory systemic lupus erythematosus: a multicenter clinical study. Arthritis Res Ther. 2014; 16(2):R79.

31. Chen $T$, Zhang $P$, Fan W, et al. Co-transplantation with mesenchymal stem cells expressing a SDF-1/HOXB4 fusion protein markedly improves hematopoietic stem cell engraftment and hematogenesis in irradiated mice. Umbilical Cord-Derived Mesenchymal Stem Cells for Hematopoietic Stem Cell Transplantation. Am J Transl Res. $2014 ; \mathbf{6}(\mathbf{6}): 691-702$.

32. van der Garde M, van Pel M, Millán Rivero JE, et al. Direct Comparison of Wharton's Jelly and Bone Marrow-Derived Mesenchymal Stromal Cells to Enhance Engraftment of Cord Blood CD34(+) Transplants. Stem Cells Dev. 2015; 24(22):2649-59.

33. Wu KH, Tsai C, Wu HP. Human Application of Ex-Vivo Expanded Umbilical Cord-Derived Mesenchymal Stem Cells: Enhance Hematopoiesis after Cord Blood Transplantation. Cell Transplant. 2013; 22(11):2041-51. doi: 10.3727/096368912X663533

34. Wu KH, Sheu JN, Wu HP. Cotransplantation of umbilical cord-derived mesenchymal stem cells promote hematopoietic engraftment in cordblood transplantation: a pilot study. Transplantation 2013; 95(5):773-77.

35. Arufe MC, De la Fuente A, Fuentes I, et al. Umbilical cord as a mesenchymal stem cell source for treating joint pathologies. World J Orthop. 2011 ; 2(6):43-50.

36. Chao Yu-Hua, Wu Han-Ping, Chan Chin-Kan, et al. Umbilical Cord-Derived Mesenchymal Stem Cells for Hematopoietic Stem Cell Transplantation. Hindawi Publishing Corporation Journal of Biomedicine and Biotechnology. 2012; 2012:759503.

37. Kim SW, Jin HL, Kang SM, et al. Therapeutic effects of late outgrowth endothelial progenitor cells or mesenchymal stem cells derived from human umbilical cord blood on infarct repair. Int J Cardiol. 2015; 203:498-507.

38. Zhao XF, Xu Y, Zhu ZY. Clinical observation of umbilical cord mesenchymal stem cell treatment of severe systolic heart failure. Shi Genet Mol Res. 2015; 14(2):301017.

39. Yan $C$, Xue G, Wu L. Differentiation of human umbilical cord mesenchymal stem cells into hepatocytes induced by rat fibrotic liver tissue extracts. Zhongguo Xiu Fu Chong Jian Wai Ke Za Zhi. 2015; 29(7):878-83.

40. Xue HL, Zeng WZ, Wu XL, et al. Clinical therapeutic effects of human umbilical cord-derived mesenchymal stem cells transplantation in the treatment of end-stage liver disease. Transplant Proc. 2015; 47(2):412-18.

41. Talaei-Khozani T, Borhani-Haghighi M, Ayatollahi M. An in vitro model for hepatocyte-like cell differentiation from Wharton's jelly derived-mesenchymal stem cells by cell-base aggregates. Gastroenterol Hepatol Bed Bench. 2015; 8(3):188-99.

42. Gu J, Gu W, Lin C, et al. Human umbilical cord mesenchymal stem cells improve the immune associated inflammatory and prothrombotic state in collagen type-II induced arthritic rats. Mol Med Rep. 2015; 12(5):7463-70.

43. Wang $X$, Wang $Y$, Gou W, et al. Role of mesenchymal stem cells in bone regeneration and fracture repair: a review. International Orthopaedics (SICOT). 2013; 37(12):2491-2498.

44. Pang X, Yang H, Peng B. Human Umbilical Cord Mesenchymal Stem Cell Transplantation for the Treatment of Chronic Discogenic Low Back Pain. Pain Physician. 2014; 17(4):E525-30.

45. Hou Z, Liu Y, Mao X, et al. Transplantation of umbilical cord and bone marrow-derived mesenchymal stem cells in a patient with relapsing-remitting multiple sclerosis. Cell Adhesion \& Migration. 2013; 7(5):404-07.

46. Cheng H, Liu X, Hua R, et al. Clinical observation of umbilical cord mesenchymal stem cell transplantation in treatment for sequelae of thoracolumbar spinal cord injury. J Transl Med. 2014; 12:253.

47. Song $L$, Zhu J, Zhang $X$, et al. BDNF-hypersecreting human umbilical cord blood mesenchymal stem cells promote erectile function in a rat model of cavernous nerve electrocautery injury. Int Urol Nephrol. 2016; 48(1):37-45.

48. Oh EJ, Kim TK, Shin JH, et al. Biologic filler using human fibroblasts and placenta extracts. J Craniofac Surg. 2011; 22(5):1557-60.

49. LiF, Chen YZ, Miao ZN, et al. Human placenta-derived mesenchymal stem cells with silk fibroin biomaterial in the repair of articular cartilage defects. Cell Reprogram. 2012; 14(4):334-41. 
50. Dominici M, Le Blanc K, Mueller I, et al. Minimal criteria for defining multipotent mesenchymal stromal cells. The International Society for Cellular Therapy position statement. Cytotherapy. 2006; 8(4):315-17.

51. Wu QL, Liu XY, Nie DM, et al. Umbilical cord blood-derived mesenchymal stem cells ameliorate graft-versus-host disease following allogeneic hematopoietic stem cell transplantation through multiple immunoregulations. J Huazhong Univ Sci Technolog Med Sci. 2015; 35(4):477-84.

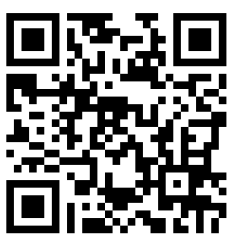

